\title{
Development of Ultrasonic-assisted Medium-speed EDM
}

\author{
XU Lin-hong, XU Qing, ZHAO Quan, CHEN Yin-di \\ Faculty of Mechanical \& Electrical Information, China University of Geosciences (Wuhan), \\ Hubei Wuhan 430074, China \\ *xulinhong@cug.edu.cn
}

Keywords: Wire Cutting; ultrasonic-assisted; cutting efficiency

\begin{abstract}
WEDM (Wire Cut Electrical Discharge Machining) is a kind of spark cutting machining processes by using the linear movable electrode (known electrode wire). With the development of science and technology and the emergence of a large number of new materials, EDM wire cutting start to face new challenges. The characteristics of these new materials such as multiphase materials processing, electrical conductivity, thermal conductivity inhomogeneity, as well as processing of large thickness of the workpiece result in low efficiency of EDM wire cutting and high frequent wire broken. Recent years, seldom research on the introduction of ultrasonic vibration into medium-speed EDM wire cutting has been done. This article aimed to analyze the influence on the cutting efficiency and workpice suface roughness by using self-made ultraconic assisted appratus on Medium-speed EDM. The results showed that ultrasonic vibration can improve the efficiency of wire electric discharge machining, and it can alao reduce the surface roughness Ra value of the workpiece. What's more, the cutting speed is higher when the direction of ultrasonic vibration and cutting direction is vertical rather than parallel.
\end{abstract}

\section{Introduction}

As a kind of new technology of material processing by using electricity and heat energy, Electrical discharge machining is different from traditional machining process with cutting tool contact directly, which remove the workpiece's material by continuous pulse discharge between electrode and workpiece. However, there are many problems such as low speed of the EDM wire cutting and higher breakage risk with electrode wire in the processing of special materials (hard alloy, titanium alloy) or much thicker workpiece. Now, ultrasonic vibration was imposed on the wire electrode to improve the discharge environment of wire electrode, cycle state of working liquid and the speed of WEDM by some researchers. But most of these researches focused on the high speed wire EDM machine tool. Where now, this paper amid to do some research on the ultrasonic vibration assisted on the Medium-speed WEDM which belong to the category of Reciprocating high speed wire EDM machine tool but with some improvement in several aspects, such as the pulse power supply, control system and movement wire system.

\section{Experiment setup and processing parameters}

\subsection{Equipment}

Ultrasonic assisted wire cutting equipment is composed by two parts: Ultrasonic assisted system and Medium-speed WEDM, where Ultrasonic assisted system composed by the ultrasonic generator, transducer, amplitude transformer and tool rod. Ultrasonic generator is also known as the ultrasonic driving power supply or ultrasonic controller, the role is to produce high power high frequency alternating current, driving transducer work. Amplitude transformer is an important part of the ultrasonic system, which will enlarge the amplitude of the transducer and pass it to the tool rod. In the ultrasonic assisted electrical discharge machining, the tool rod is the medium between the amplitude transformer and the electrode wire with high speed reciprocating motion to attach the ultrasonic 
vibration to the wire electrode. In this case, the vibration state of the electrode wire is changed. The schematic diagram of ultrasonic-assisted WEDM is shown in Figure 1.
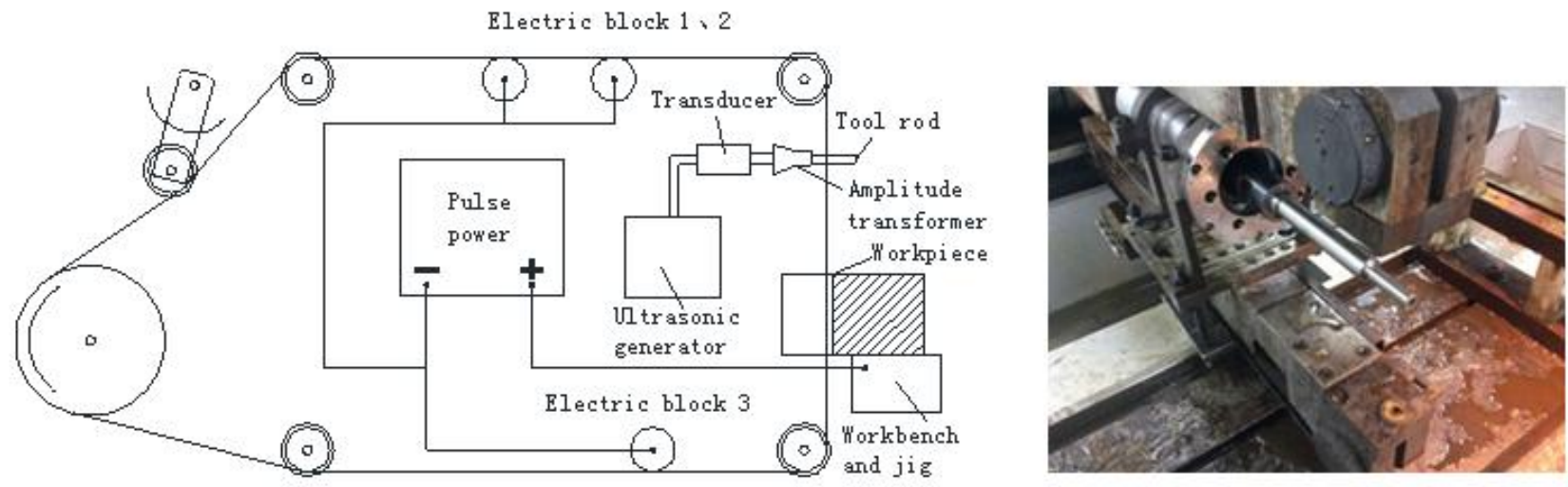

Fig. 1 Schematic diagram (left) and apparatus (right)

Equipment of Medium-speed WEDM in this work is BM500D produced by Suzhou Baoma NC Equipment Co., Ltd with BMXP control system. The Ultrasonic vibration assisting system is composed by Chen Gong V6.0 ultrasonic generator, Chen Gong YP5020-4D transducer, An amplitude transformer with step shape made of material $45^{\#}$ adopted, and tool rod made of $45^{\#}$. Length of the tool rod is determined as $135 \mathrm{~mm}$ by which a longitudinal vibration can be produced and transmitted amplitude of amplitude transformer to the electrode wire stably through simulated by Ansys and experimental verification. This set of system is mounted on the beam of the Medium-speed WEDM (shown in Figure 1). Since the power of the ultrasonic generator is $1 \mathrm{Kw}$ with an adjustable range $30 \% \sim 90 \%$, the experiment adopt $60 \%$ power. The working frequency is $20.1 \mathrm{KHz}$ with tool rod on and the amplitude of the tool rod can be measured as $22 \mu \mathrm{m}$.

\subsection{Experiment design}

In this paper, Mo electrode filament with $0.18 \mathrm{~mm}$ is adopted with tension force $9.8 \mathrm{~N}$ and $220 \mathrm{~mm}$ span. While 45\# and Cr12 was chosen as workpiece material for cutting experiment. 45\# steel is a kind of high carbon steel with excellent properties. And $\mathrm{Cr} 12$ is a high carbon and high chromium steel widely used in the manufacture of cold die and punch, gauge, a drill sleeve, wire drawing die, stamping die and drawing die mold. Two dimensions of sample $10 \mathrm{~mm} \times \mathrm{mm} \times 50 \mathrm{~mm}$ and $10 \mathrm{~mm} \times 6$ $\mathrm{mm} \times 80 \mathrm{~mm}$ is used in this work.

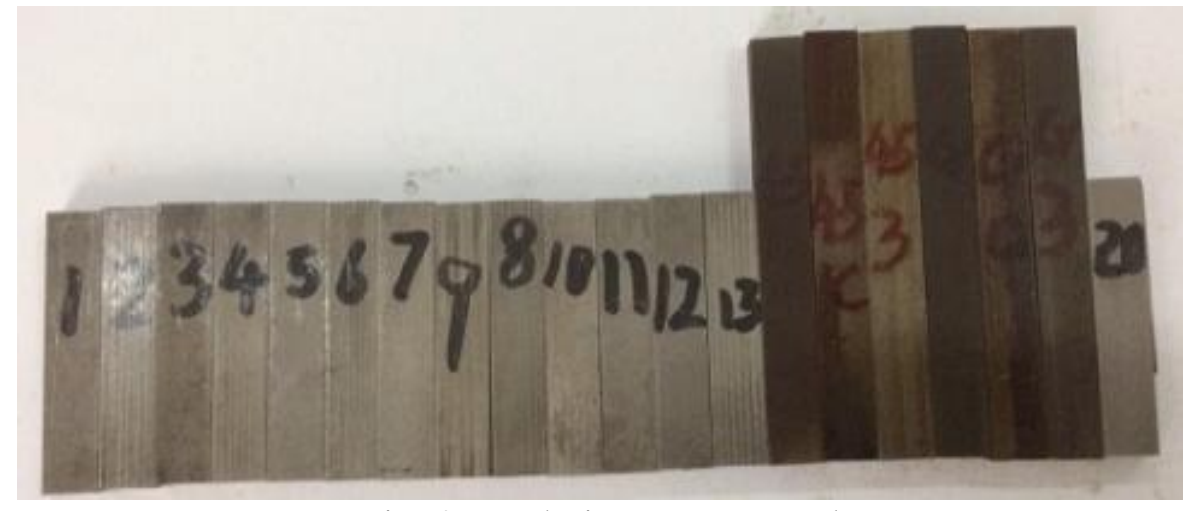

Fig. 2 Workpieces processed

To compare the cutting result with or without ultrasonic vibration, two groups of experiments was designed. First, two material with same thickness, $80 \mathrm{~mm}$, was cut by different ultrasonic vibration types with parallel and perpendicular to the feed direction or without ultrasonic vibration assisted receptively. The result of first group will focus on the cutting speed. Second, two materials with $50 \mathrm{~mm}$ thickness was cut in the emulsified oil and two materials with $80 \mathrm{~mm}$ thickness was cut in BM2water based fluid by different ultrasonic vibration types with parallel and perpendicular to the feed direction or without ultrasonic vibration assisted receptively (shown in Fig.3). The result of the second group will focus on the surface roughness of workpiece cut. The surface can be cut by several 
times to improve surface roughness. And the finishing allowance is set as $0.04 \mathrm{~mm}, 0.02 \mathrm{~mm}$ and $0 \mathrm{~mm}$ for three times cut. And pulse width, pulse distance are the control parameters during material cutting.

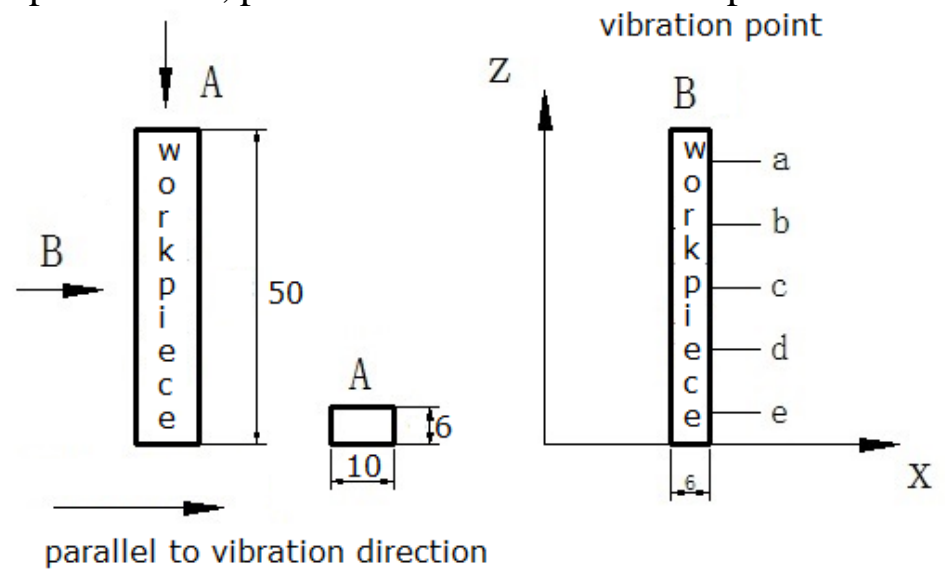

Fig. 3 Vibration direction under work condition

\section{Result and analysis}

\subsection{Cutting efficiency}

As can be seen from the Table 1, the cutting efficiency can be improved when the ultrasonic vibration attached to the wire. At same time, with the increase of the pulse width, the cutting speed of $45 \#$ increases gradually. The improvement of efficiency can reach $22.9 \%$ when the pulse width is at $72 \mu \mathrm{m}$. Table 1 also shows that machining speed with ultrasonic vibration direction vertical to the feed direction is higher slightly than those parallel to the feed direction, which mean ultrasonic vibration can influence the product of peak current and peak voltage of machine tool.

Table 1 Cutting efficiency under different conditions

\begin{tabular}{|c|c|c|c|c|c|}
\hline $\begin{array}{c}\text { Workpiece } \\
\text { material }\end{array}$ & $\begin{array}{l}\text { Thickness } \\
(\mathrm{mm})\end{array}$ & $\begin{array}{l}\text { Cutting } \\
\text { times }\end{array}$ & $\begin{array}{l}\text { Without vibration assisted } \\
\qquad\left(\mathrm{mm}^{2} / \mathrm{min}\right)\end{array}$ & $\begin{array}{c}\text { Vibration direction } \\
\text { vertical to feed } \\
\left(\mathrm{mm}^{2} / \mathrm{min}\right)\end{array}$ & $\begin{array}{l}\text { Vibration direction } \\
\text { parallel to feed } \\
\left(\mathrm{mm}^{2} / \mathrm{min}\right)\end{array}$ \\
\hline \multirow{5}{*}{$45 \#$} & \multirow{4}{*}{50} & once & 31.71 & 35.01 & 32.32 \\
\hline & & \multirow{3}{*}{ Three cuts } & 41.90 & 46.86 & 43.27 \\
\hline & & & 96.79 & 99.77 & 107.99 \\
\hline & & & 55.42 & 57.14 & 58.37 \\
\hline & 80 & once & 18.58 & 20.76 & 19.18 \\
\hline \multirow{5}{*}{ Cr12 } & \multirow{4}{*}{50} & once & 30.25 & 31.02 & 33.78 \\
\hline & & \multirow{3}{*}{ Three cuts } & 54.35 & 56.39 & 57.01 \\
\hline & & & 112.47 & 115.38 & 124.69 \\
\hline & & & 83.8 & 88.5 & 87.2 \\
\hline & 80 & once & 16.625 & 17.46 & 17.09 \\
\hline
\end{tabular}

\subsection{Workpiece surface roughness}

The surface roughness of workpiece processed is shown in Figure 4. Among these, figure a is for 45\# 50mm thickness after once cut; figure b is Cr12 50mm thickness after once cut; figure c 45\# $50 \mathrm{~mm}$ thickness after three times cut; figure $\mathrm{d}$ Cr12 50mm thickness after three times cut; figure e 45\# $80 \mathrm{~mm}$ thickness after once cut; figure $\mathrm{f} \mathrm{Cr} 1280 \mathrm{~mm}$ thickness after once cut. The measure point is shown in figure 6. Emulsified oil was adopted for lubrication for all workpieces with $50 \mathrm{~mm}$ thickness while BM2 water based fluid adopted for all workpieces with $80 \mathrm{~mm}$ thickness. The surface roughness of workpiece was measured by using 


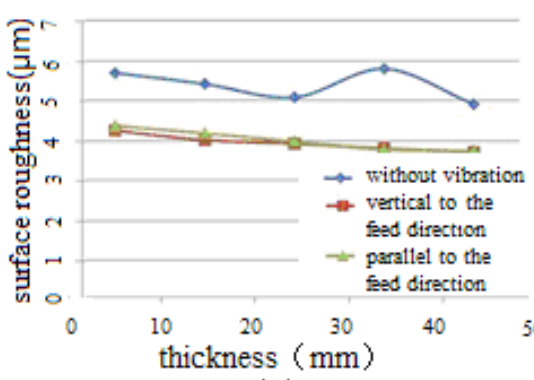

(a)

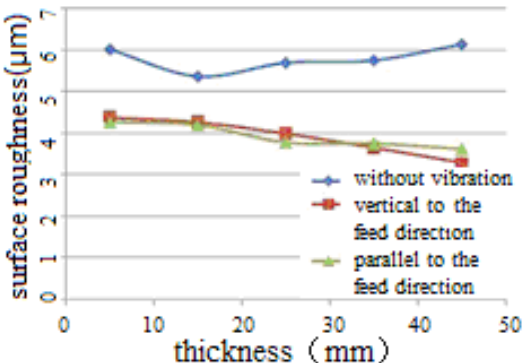

(d)

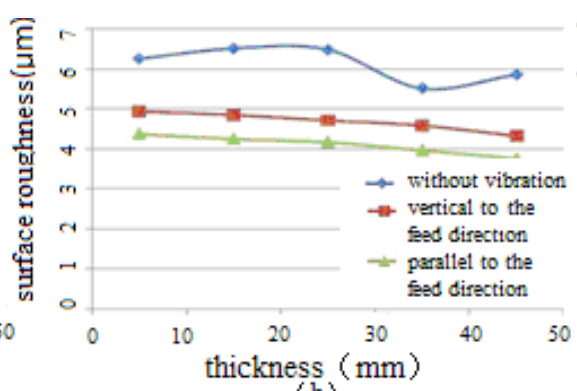

(b)

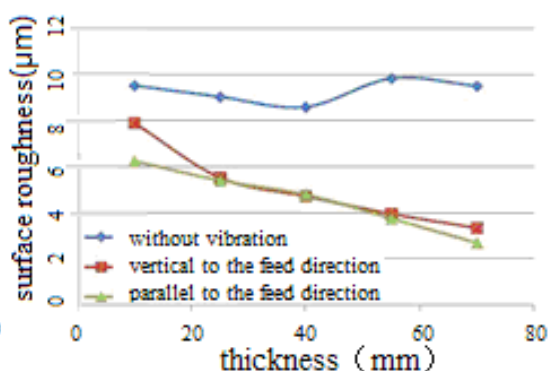

(e)

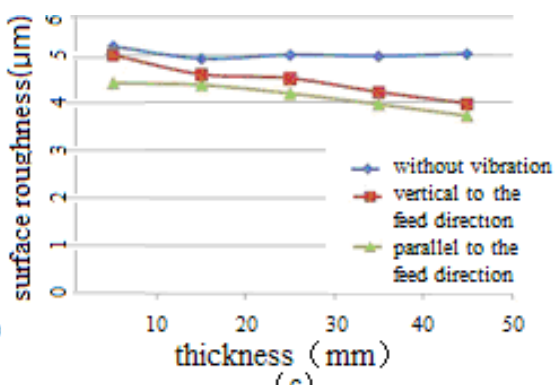

(c)

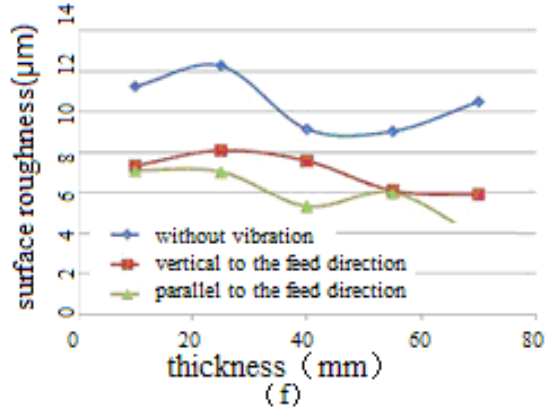

Fig. 4 Workpiece surface roughness processed (blue line for cutting without vibration, red one for vibration direction vertical to feed and green one for vibration direction parallel to feed)

From Figure 4, we can find that Ra values of both 45\# and Cr12 workpiece surface reduced at different degree which mean surface quality improved accordingly as assisted by ultrasonic vibration. When the ultrasonic vibration direction is vertical to the feed direction, the interruption of partial discharge pulse will occur due to the vibration between the electrode filament and the surface of the workpiece, which will result nonuniform discharge on the workpiece surface and formation of pit with different size and shape. Fortunately, when ultrasonic vibration direction is parallel to the feed direction, a certain distance between the electrode filament and the workpiece surface can be maintained to promise better surface quality obtained than that in vertical case.

Besides this, when water based fluid BM2 applied, the roughness value and rusting degree can vary on the workpiece surface processed after ultrasonic vibration introduced. The rusting area on the surface cut under ultrasonic vibration assisted is much smaller than that without ultrasonic vibration assisted.

\section{Conclusions}

The conclusions can be made through the above cutting experiment with or without self-made ultrasonic vibration assisting WEDM appartus as follows: the cutting efficiency and workpiece surface roughness can all be improved in certain degree with ultrasonic vibration assisting, and the influence of different vibration direction relative to feed direction can not to be neglected.

\section{References}

[1] Pandit. S. M, Rajurkar K. P, Data dependent systems approach to EDM process modeling from surface roughness profiles [C].Annals of the CIRP,1980,29: 107-112.

[2] V.S.R. Murti, P.K. Philip, An analysis of the debris in ultrasonic-assisted electrical discharge machining.Wear.1987, 117 (2) :12 16

[3]D. kremer, J.L. Lebrun, B. Hosari, A. Moisan, Effeets of ultrasonic vibrations on the performances in EDM.Annals of the CIRP.1989， 38 (1):199—202

[4]Harry Moser, It's Time to EDM Tooling \& Production [J].1998, (1):65-70.

[5]Y. S Liao, J.C. Woo, Design of a fuzzy controller fou the adaptive control of WEDM process [J]. International Journal of machine Tools \& Manufacture 40(2000): 2293-2307. 